\title{
A Multi-Domain Bivariate Approach for Mixed Convection in a Casson Nanofluid with Heat Generation
}

\author{
Ibukun Sarah OYELAKIN ${ }^{1}$, Sabyasachi MONDAL ${ }^{2, *}$, \\ Precious SIBANDA ${ }^{1}$ and Sandile Sydney MOTSA ${ }^{3}$
}

\author{
${ }^{I}$ School of Mathematics, Statistics and Computer Science University of KwaZulu-Natal, \\ Scottsville 3209, South Africa \\ ${ }^{2}$ Department of Mathematics, Amity University, Kolkata, Newtown-700135, West Bengal, India \\ ${ }^{3}$ Department of Mathematics, University of Swaziland, Kwaluseni, Swaziland
}

('Corresponding author's e-mail: sabya.mondal.2007@gmail.com)

Received: 14 September 2016, Revised: 13 July 2018, Accepted: 21 August 2018

\begin{abstract}
We study the mixed convection flow of a Casson nanofluid past a permeable moving flat plate with heat generation, chemical reaction and viscous dissipation effects in the presence of thermo and thermal diffusion effects. The fluid model described assumes the nanoparticle flux at the boundary is zero, and suction effects on the velocity boundary are accounted for. The system of partial differential equations obtained is solved using a multi-domain bivariate quasi-linearization method with a detailed description of the numerical method of solution. The effects of various fluid parameters on the velocity, temperature, and nanoparticle concentration profiles, as well as on the local skin friction, and heat and mass transfer coefficients, are discussed in detail.
\end{abstract}

Keywords: Casson nanofluid, viscous dissipation, suction/injection, multi-domain bivariate spectral quasi-linearization method

\section{Introduction}

Mixed convection refers to the combination of natural and forced convection mechanisms acting together to significantly influence heat transfer processes. Mixed convection occurs in many industrial processes; the major ones include, for example, nuclear reactor technology [1]. Natural and forced convection are dependent on the laminar flow regime; the magnitude of the temperature driving force is reflected in the thermal Grashof number and the nature of the fluid flow. Mixed convection studies on permeable surfaces have been carried out by, among others, Isa et al. [2], on a steady boundary layer flow of a Casson fluid. Hayat et al. [3] considered a 2-dimensional steady stagnation point flow of an incompressible Casson fluid over a stretching surface with convective boundary condition. Sharada and Shankar [4] studied the steady laminar incompressible magnetohydrodynamic flow of a Casson fluid over an exponentially stretching surface with Soret and Dufour effects, thermal radiation, and chemical reaction effect using the Keller box method for the solution of the model equations. The influence of the convective boundary condition on mixed convection boundary layer flow of a Casson nanofluid over a stretching sheet with a heat source/sink was studied by Hayat et al. [5].

A chemical reaction may be either a homogeneous or heterogenous process, depending on its occurrence at an interface or in a single-phase volume reaction. The influence of chemical reaction on coupled heat and mass transfer in fluid flow is important in certain processes, such as hydrometallurgical industries, power and cooling, and drying, among many others. Due to the perceived importance of heat and mass transfer processes combined with a chemical reaction, some attention has been given to research in 
http://wjst.wu.ac.th

this area in recent years. Kandasamy et al. [6] presented an analysis of steady laminar magnetohydromagnetic flow with heat and mass transfer from a stagnation point on a wedge plate embedded in a porous medium. The studied considered the effects of variable viscosity, thermal stratification, a first order chemical reaction, and suction or injection. Makanda et al. [7] studied the magnetohydrodynamic free convection flow of a Casson fluid from a horizontal circular cylinder with partial slip in a non-Darcy porous medium with viscous dissipation and radiative effects. In the work, the coupled nonlinear governing partial differential equations were solved using a bi-variate quasi-linearization method and validated using a successive linearization method and MATLAB's 'bvp4c'. Aslefallah et al. [8-11] used the finite difference theta method to solve some space and poisson fractional equations.

Diffusion-thermo, or Dufour, effect is the energy flux which occurs not only by temperature gradients, but also due to the effects of concentration gradient, while the thermal diffusion, or Soret, effect is as a result of mass flux occurring due to the temperature gradient. Thermal diffusion is utilized for isotope or species separation in a fluid flow. Kafoussias and Williams [12] considered mixed convection and mass transfer in a viscous incompressible fluid over an isothermal semi-infinite vertical flat plate. Their work investigated thermal-diffusion and diffusion-thermo effects when the fluid viscosity varied with the temperature. Chamkha and El-Kabeir [13] studied the unsteady laminar and coupled heat and mass transfer of magnetohydrodynamic mixed convective boundary-layer flow of an electrically conducting fluid over an impulsively stretched vertical surface in an unbounded quiescent fluid with aiding external flow in the presence of a transverse magnetic field, homogenous chemical reaction, and Soret and Dufour effects. The governing equations were non-dimensionalized using the Williams and Rhyne transformation, and the resulting non-similar equations solved using the standard implicit finite difference method. They concluded that, as the Dufour number decreased, the skin friction coefficient, the Nusselt number, and the Sherwood number increased, while the opposite trend was observed for the Soret number.

Due to the low thermal conductivity of most Newtonian fluids, nano-sized particles are now routinely added to enhance the thermophysical properties of common fluids and, thus, improve heat transfer processes. This innovation is due to Choi [14], who proposed the suspension of nanoparticles to these so-called base fluids. Buongiorno [15] explained 7 possible mechanisms for the enhancement of the heat transfer process in nanofluids, and concluded by noting that the most important of these mechanisms are thermophoresis and the Brownian motion of the nanoparticles. A model was developed to take into account of these processes. Kuznetsov and Nield [16] revised the earlier model by assuming that the nanoparticles flux at the boundary is zero, so that the nanoparticle volume fraction at the boundary is passive rather than actively controlled. Fakour et al. [17] studied the steady fully-developed mixed convection flow of a nanofluid filled in a vertical channel, which is driven by an external pressure gradient and a buoyancy force. Khan et al. [18] discussed 3-dimensional incompressible flow of a nanofluid induced due to a surface stretched nonlinearly in 2 lateral directions, and considered the passive control of nanoparticle volume fraction at the wall. Recent publications on Casson nanofluid can be found in [19,22].

In this study, mixed convection in a Casson nanofluid flow past a permeable moving flat plate is studied. We assume the presence of a chemical reaction, heat generation, and diffusion-thermo and thermal diffusion effects. A second innovation in this paper is the use of multi-domain bivariate quasi-linearization method to find numerical solutions of the nonlinear differential equations that describe the fluid flow model. This method of solution is accurate and has low CPU times. Another important characteristic of the multidomain bivariate quasi-linearization method is that it allows for the prediction of heat and mass transfer coefficients for large values of the independent variable. The results were validated via a comparative analysis with existing literature values for certain parameter values.

\section{Mathematical formulation}

Consider mixed convection in steady 2-dimensional boundary layer flow of a Casson nanofluid over a permeable flat plate moving with velocity $U_{w}$ and free stream velocity $U_{\infty}$. The physical co-ordinates $x$ and $y$ are chosen, such that the $x$-axis is along the plate in the vertical direction, the $y$-axis is normal to it, and $u$ and $v$ are the velocity components along the $x$ and $y$ directions, respectively; see Figure 1. It is assumed that the plate is maintained at a uniform and constant wall temperature $T_{w}$ and the temperature $T_{w}$ 
http://wjst.wu.ac.th

is greater than the ambient temperature $T_{\infty}$ at any particular point in the boundary layer. The Casson fluid and the nanoparticles are assumed to be in thermal equilibrium without slip. The nanoparticle flux at the boundary is assumed to be zero and viscous dissipation is considered to be important in the analysis.

The rheological equation of state for an isotropic and incompressible flow of a Casson fluid is expressed (see [23]) as;

$\tau_{i j}= \begin{cases}2\left(\mu_{B}+\frac{p_{y}}{\sqrt{2 \pi}}\right) e_{i j} & \text { if } \pi>\pi_{c} \\ 2\left(\mu_{B}+\frac{p_{y}}{\sqrt{2 \pi_{c}}}\right) e_{i j} & \text { if } \pi<\pi_{c}\end{cases}$

where $e_{i j}$ is the $(i, j)$ th component of the rate of strain tensor, $\tau_{i j}$ is the $(i, j)$ th component of the stress tensor, $\mu_{B}$ is the Casson coefficient of viscosity, $\pi=e_{i j} e_{i j}$ is the product of the rate of strain tensor with itself, $\pi_{c}$ is the critical value of the product of the rate of strain tensor with itself, and $p_{y}$ is the yield stress of the fluid.

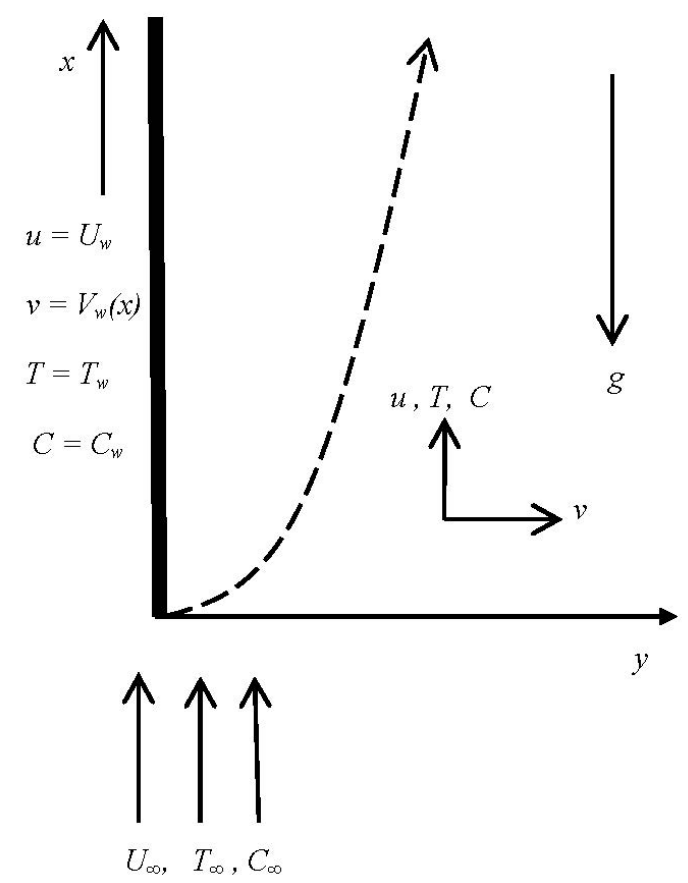

Figure 1 Schematic diagram of flow problem under consideration.

Under the Boussinesq and boundary layer approximations, the conservation equations are given as;

$\frac{\partial u}{\partial x}+\frac{\partial v}{\partial y}=0$

$u \frac{\partial u}{\partial x}+v \frac{\partial u}{\partial y}=v\left(1+\frac{1}{\beta}\right) \frac{\partial^{2} u}{\partial y^{2}}+g \beta_{T}\left(T-T_{\infty}\right)+g \beta_{C}\left(C-C_{\infty}\right)$,

$u \frac{\partial T}{\partial x}+v \frac{\partial T}{\partial y}=\alpha_{m} \frac{\partial^{2} T}{\partial y^{2}}+\frac{v}{c_{p}}\left(1+\frac{1}{\beta}\right)\left(\frac{\partial u}{\partial y}\right)^{2}+Q_{0}\left(T-T_{\infty}\right)+\tau D_{B} \frac{\partial C}{\partial y} \frac{\partial T}{\partial y}+\tau \frac{D_{T}}{T_{\infty}}\left(\frac{\partial T}{\partial y}\right)^{2}+\frac{D_{m} k_{T}}{c_{s} c_{p}} \frac{\partial^{2} C}{\partial y^{2}}$, 
http://wjst.wu.ac.th

$u \frac{\partial C}{\partial x}+v \frac{\partial C}{\partial y}=D_{B} \frac{\partial^{2} C}{\partial y^{2}}-k_{c}\left(C-C_{\infty}\right)+\frac{D_{T}}{T_{\infty}} \frac{\partial^{2} T}{\partial y^{2}}+\frac{D_{m} k_{T}}{T_{m}} \frac{\partial^{2} T}{\partial y^{2}}$

where $u$ and $v$ are the velocities in the $x$-and $y$-directions, respectively, $\beta=\mu_{B} \frac{\sqrt{2 \pi_{c}}}{p_{y}}$ is the Casson fluid parameter, $v$ is the kinematic viscosity, $g$ is the acceleration due to gravity, $\beta_{T}$ and $\beta_{C}$ are the coefficient of thermal diffusivity and concentration expansion, respectively, $\alpha_{m}$ is the thermal diffusivity, $c_{p}$ is the specific heat at constant pressure, $Q_{o}$ is the heat generation constant, $\tau=(\rho c)_{p} /(\rho c)_{f}$ is the ratio of the heat capacity of the nanoparticle material and the heat capacity of the Casson fluid, $D_{B}$ is the Brownian diffusion coefficient, $D_{T}$ is the thermophoretic diffusion coefficient, $D_{m}$ is the mass diffusivity, $k_{T}$ is the thermal diffusivity, $c_{S}$ is the concentration susceptibility, $k_{c}$ is the chemical reaction rate, and $T_{m}$ is the mean temperature.

The associated boundary conditions are;

$$
\begin{array}{ll}
u=U_{w}, \quad v=V_{w}, \quad T=T_{w}, \quad D_{B} \frac{\partial C}{\partial y}+\frac{D_{T}}{T_{\infty}} \frac{\partial T}{\partial y}=0, \quad \text { aty }=0 \\
u \rightarrow U_{\infty}, \quad T \rightarrow T_{\infty}, \quad C \rightarrow C_{\infty}, \quad \text { asy } \rightarrow \infty .
\end{array}
$$

The variable plate surface permeability function is given as;

$$
V_{w}(x)=-\frac{f_{w}}{2} \sqrt{\frac{U v}{x}}
$$

where $U=U_{w}+U_{\infty}, f_{w}$ is a constant, $f_{w}>0$ represents suction effects at the plate surface, $f_{w}<0$ is injection, and $f_{w}=0$ represents an impermeable surface. For the continuity equation to be satisfied, introduce the stream function $\psi$ approach, such that;

$u=\frac{\partial \psi}{\partial y}, \quad v=-\frac{\partial \psi}{\partial x}$

The system of non-linear partial differential equations are reduced by introducing the following non-dimensional variables;

$\psi=(U v x)^{\frac{1}{2}} f(\zeta, \eta), \quad \eta=y\left(\frac{U}{v x}\right)^{\frac{1}{2}}, \quad \zeta=\left(\frac{U x}{v}\right)^{\frac{1}{2}}, \quad \frac{T-T_{\infty}}{T_{w}-T_{\infty}}=\vartheta(\zeta, \eta), \frac{C-C_{\infty}}{C_{w}-C_{\infty}}=\varphi(\zeta, \eta)$,

where $f(\zeta, \eta), \vartheta(\zeta, \eta)$ and $\varphi(\zeta, \eta)$ are the non-dimensional velocity, temperature, and concentration, respectively. Using the transformations defined in (10), Eqs. (2) - (5), together with the boundary conditions in (6) and (7), are transformed to the following non-dimensional form;

$$
\begin{aligned}
& \left(1+\frac{1}{\beta}\right) f^{\prime \prime \prime}+\frac{1}{2} f f^{\prime \prime}+\lambda\left(\vartheta+N_{1} \varphi\right)=\frac{\zeta}{2}\left(f^{\prime} \frac{\partial f^{\prime}}{\partial \zeta}-f^{\prime \prime} \frac{\partial f}{\partial \zeta}\right), \\
& \frac{1}{P r} \vartheta^{\prime \prime}+\frac{1}{2} f \vartheta^{\prime}+E c\left(1+\frac{1}{\beta}\right) f^{\prime 2}+H e \zeta^{2} \vartheta+N_{b} \vartheta^{\prime} \varphi^{\prime}+N_{t} \vartheta^{\prime 2}+D_{f} \varphi^{\prime \prime}=\frac{\zeta}{2}\left(f^{\prime} \frac{\partial \vartheta}{\partial \zeta}-\vartheta^{\prime} \frac{\partial f}{\partial \zeta}\right), \\
& \varphi^{\prime \prime}+\frac{L e}{2} f \varphi^{\prime}-L e \zeta^{2} R \varphi+\frac{N_{t}}{N_{b}} \vartheta^{\prime \prime}+L e S r \vartheta^{\prime \prime}=L e \frac{\zeta}{2}\left(f^{\prime} \frac{\partial \varphi}{\partial \zeta}-\varphi^{\prime} \frac{\partial f}{\partial \zeta}\right) .
\end{aligned}
$$

The transformed boundary conditions are;

$$
f(\zeta, 0)+\zeta \frac{\partial f(\zeta, 0)}{\partial \zeta}=f_{w}, f^{\prime}(\zeta, 0)=1-r, \vartheta(\zeta, 0)=1, N_{b} \varphi^{\prime}(\zeta, 0)+N_{t} \vartheta^{\prime}(\zeta, 0)=0
$$


$f^{\prime}(\zeta, \infty)=r, \quad \vartheta(\zeta, \infty)=0, \quad \varphi(\zeta, \infty)=0$,

where $G_{r}=\frac{g \beta_{T}\left(T_{w}-T_{\infty}\right) x^{3}}{v^{2}}$ and $G_{c}=\frac{g \beta_{C}\left(C_{w}-C_{\infty}\right) x^{3}}{v^{2}}$ are the thermal and solutal Grashof numbers, respectively, $\lambda=G_{r} / R e_{x}^{2}$ and $\lambda^{*}=G_{c} / R e_{x}^{2}$ are the buoyancy parameters, $N_{1}=\lambda^{*} / \lambda$ is the ratio of the buoyancy forces, $R e_{x}=U x / v$ is the local Reynolds number, $r=U_{\infty} / U$ is the velocity ratio parameter, $\operatorname{Pr}=v / \alpha_{m}$ is the Prandtl number, $E c=U^{2} / c_{p}\left(T_{w}-T_{\infty}\right)$ is the Eckert number, $H e=Q_{0} v / U^{2}$ is the heat generation parameter, $N_{b}=\tau D_{B}\left(C_{w}-C_{\infty}\right) / v$ is the Brownian motion parameter, $N_{t}=\tau D_{T}\left(T_{w}-\right.$ $\left.T_{\infty}\right) / v T_{\infty}$ is the thermophoresis parameter, $D_{f}=\frac{D_{m} k_{T}\left(C_{w}-C_{\infty}\right)}{c_{s} C_{p} v\left(T_{w}-T_{\infty}\right)}$ is the Dufour number, $L e=v / D_{B}$ is the Lewis number, $\mathrm{R}=\frac{k_{c} v}{U^{2}}$ is the chemical reaction parameter, and $S r=\frac{D_{m} k_{T}\left(T_{w}-T_{\infty}\right)}{v\left(C_{w}-C_{\infty}\right) T_{m}}$ is the Soret number.

\section{Skin friction, rate of heat and mass transfer}

The local wall shear stress denoted by $\tau_{w}$ and the local surface heat flux $q_{w}$ are evaluated, respectively, from the following;

$\tau_{w}=\left(\mu_{B}+\frac{p_{y}}{\sqrt{2 \pi_{c}}}\right)\left(\frac{\partial u}{\partial y}\right)_{y=0}, \quad q_{w}=-k\left(\frac{\partial T}{\partial y}\right)_{y=0}$.

The dimensionless skin friction coefficient denoted by $C_{f x}$ and the local Nusselt number $N u_{x}$ are defined as;

$C_{f x}=\frac{\tau_{w x}}{\frac{1}{2} \rho U^{2}}, \quad N u_{x}=\frac{x q_{w}}{k\left(T_{w}-T_{\infty}\right)^{\prime}}$

Hence, using the similarity variables given in (11), we obtain;

$R e_{x}^{\frac{1}{2}} C_{f x}=2\left(1+\frac{1}{\beta}\right) f^{\prime \prime}(\zeta, 0), \quad R e_{x}^{-\frac{1}{2}} N u_{x}=-\vartheta^{\prime}(\zeta, 0)$.

\section{Method of solution}

Multi-domain bivariate spectral quasi-linearization method(MD-BSQLM)

In this section we first provide a description of the MD-BSQLM for solving systems of differential equations. The multi-domain approach has been used previously to solve systems of first order initial value problems that model chaotic systems (see Motsa et al. [24]). In this study, the method is extended to the nonlinear system of Eqs. (11) - (13). It is worth to noting that the multidomain approach is applied in the $\zeta$ direction only. To apply the concept to our system of equations, we let $\zeta \in \Lambda$, where $\Omega=[0, T]$ and decompose $\Lambda$ into $p$ non-overlapping intervals defined by;

$\Lambda_{m}=\left[\zeta_{m-1}, \zeta_{m}\right], \quad m=1,2, \cdots, p$, with $0=\zeta_{0}<\zeta_{1}<\zeta_{2}<\cdots<\zeta_{p}=T$.

In the framework of the multi-domain approach, the partial differential equations are solved independently on each sub-domain using the initial condition for the solution in the first interval. When the solution has been computed, the new solution at the right hand boundary of the first interval is then used as an initial solution for the second interval. At the $m$ th interval, the computed solution at the right hand boundary of the $m-1$ th interval is used as the initial solution. If we denote $\zeta$ in the interval $\Omega_{m}$ by $\zeta_{(m)}$ and the solution of Eqs. (11) - (13) by $f^{(m)}\left(\zeta_{(m)}, \eta\right), \vartheta^{(m)}\left(\zeta_{(m)}, \eta\right)$, and $\varphi^{(m)}\left(\zeta_{(m)}, \eta\right)$, respectively, then, in the first interval $\left[\zeta_{0}, \zeta_{1}\right]$, the solutions;

$f^{(1)}(\zeta, \eta), \quad \vartheta^{(1)}(\zeta, \eta), \quad \varphi^{(1)}(\zeta, \eta)$, 
http://wjst.wu.ac.th

are obtained subject to the "initial" conditions;

$f^{(1)}(0, \eta), \quad \vartheta^{(1)}(0, \eta), \quad \varphi^{(1)}(0, \eta)$

At each interval $\left[\zeta_{m-1}, \zeta_{m}\right],(m \geq 2)$, the continuity conditions;

$f^{(m)}\left(\zeta_{m-1}, \eta\right)=f^{(m-1)}\left(\zeta_{m-1}, \eta\right), \vartheta^{(m)}\left(\zeta_{m-1}, \eta\right)=\vartheta^{(m-1)}\left(\zeta_{m-1}, \eta\right)$,

$\varphi^{(m)}\left(\zeta_{m-1}, \eta\right)=\varphi^{(m-1)}\left(\zeta_{m-1}, \eta\right)$,

This process is repeated to generate a sequence of solutions;

$f^{(m)}(\zeta, \eta), \vartheta^{(m)}(\zeta, \eta), \varphi^{(m)}(\zeta, \eta)$.

The next step is to linearize the nonlinear system of equations.

\section{Linearization}

The nonlinear partial differential equations are linearized using the quasi-linearization (QLM) technique (see [25-27]). We derive the scheme by linearizing the nonlinear components of the governing equations using Taylor series expansion. It is assumed in the linearization technique that the difference between the value of the unknown function at the current iteration level denoted by $e+1$ and the value at the previous iteration level denoted by $e$ is small. Applying the QLM technique to Eqs. (11) - (13) gives the following;

$$
\begin{aligned}
& a_{0, e} f_{e+1}^{\prime \prime \prime}(m) \\
& b_{0, e} \vartheta_{e+1}^{\prime \prime(m)}+a_{1, e} f_{e+1}^{\prime \prime(m)}+b_{1, e} \vartheta_{e+1}^{\prime(m)}+b_{2, e} \vartheta_{e+1}^{\prime(m)}+a_{3, e} f_{e+1}^{(m)}+b_{4, e} \frac{\partial f_{e+1}^{\prime(m)}}{\partial \zeta}+b_{5, e} \frac{\partial f_{e+1}^{(m)}}{\partial \zeta}+a_{6, e} \vartheta_{e+1}^{(m)}+a_{7, e} \varphi_{e+1}^{(m)}=b_{1, e} f_{e+1}^{\prime \prime(m)}+b_{5, e} f_{e+1}^{\prime(m)}+b_{6, e} f_{e+1}^{(m)}+b_{7, e} \frac{\partial f_{e+1}^{(m)}}{\partial \zeta}+ \\
& b_{8, e} \varphi_{e+1}^{\prime \prime(m)}+b_{9, e} \varphi_{e+1}^{\prime(m)}=R_{2, e}, c_{0, e} \varphi_{e+1}^{\prime \prime(m)}+c_{1, e} \varphi_{e+1}^{\prime(m)}+c_{2, e} \varphi_{e+1}^{(m)}+c_{3, e} \frac{\partial \varphi_{e+1}^{(m)}}{\partial \zeta}+c_{4, e} f_{e+1}^{\prime(m)}+c_{5, e} f_{e+1}^{(m)}+ \\
& c_{6, e} \frac{\partial f_{e+1}^{(m)}}{\partial \zeta}+c_{7, e} \vartheta_{e+1}^{\prime \prime(m)}=R_{3, e}
\end{aligned}
$$

where the variable coefficients $a_{i, e}, b_{i, e}, c_{i, e}$, and $d_{i, e}(i=1,2,3, \ldots)$ are known from previous calculations and are given by;

$$
\begin{aligned}
& a_{0, e}=1+\frac{1}{\beta}, \quad a_{1, e}=\left(\frac{1}{2}\right)\left(f_{e}^{(m)}+\zeta \frac{\partial f_{e}^{(m)}}{\partial \zeta}\right), a_{2, e}=-\left(\frac{1}{2}\right) \zeta \frac{\partial f_{e}^{\prime(m)}}{\partial \zeta}, \quad a_{3, e}=\left(\frac{1}{2}\right) f_{e}^{\prime \prime(m)}, \\
& a_{4, e}=-\left(\frac{1}{2}\right) \zeta f_{e}^{\prime(m)}, \quad a_{5, e}=\left(\frac{1}{2}\right) \zeta f_{e}^{\prime \prime(m)}, a_{6, e}=\lambda, \quad a_{7, e}=\lambda N_{1}, \\
& b_{0, e}=\frac{1}{P r}, \quad b_{1, e}=\left(\frac{1}{2}\right)\left(f_{e}^{(m)}+\zeta \frac{\partial f_{e}^{(m)}}{\partial \zeta}\right)+N_{b} \varphi_{e}^{\prime(m)}+2 N_{t} \vartheta_{e}^{\prime(m)}, \quad b_{2, e}=H e \zeta^{2}, \\
& b_{3, e}=-\left(\frac{1}{2}\right) \zeta f_{e}^{\prime(m)}, \quad b_{4, e}=2 E c\left(1+\frac{1}{\beta}\right) f_{e}^{\prime \prime(m)}, \quad b_{5, e}=-\left(\frac{1}{2}\right) \zeta \frac{\partial \vartheta_{e}^{(m)}}{\partial \zeta}, \\
& b_{6, e}=\left(\frac{1}{2}\right) \vartheta_{e}^{\prime(m)}, \quad b_{7, e}=\left(\frac{1}{2}\right) \zeta \vartheta_{e}^{\prime(m)}, b_{8, e}=D_{f}, \quad b_{9, e}=N_{b} \vartheta_{e}^{\prime(m)}, \\
& c_{0, e}=1, \quad c_{1, e}=\left(\frac{1}{2}\right) L e\left(f_{e}^{(m)}+\zeta \frac{\partial f_{e}^{(m)}}{\partial \zeta}\right), \quad c_{2, e}=-L e R \zeta^{2}, \quad c_{3, e}=-\left(\frac{1}{2}\right) L e \zeta f_{e}^{\prime(m)}, \\
& c_{4, e}=-\left(\frac{1}{2}\right) L e \zeta \frac{\partial \varphi_{e}^{(m)}}{\partial \zeta}, \quad c_{5, e}=\left(\frac{1}{2}\right) L e \varphi_{e}^{\prime(m)}, \quad c_{6, e}=\left(\frac{1}{2}\right) L e \zeta \varphi_{e}^{\prime(m)}, \quad c_{7, e}=\frac{N_{t}}{N_{b}}+L e S r .
\end{aligned}
$$


http://wjst.wu.ac.th

and the right hand side $R_{i, e}(i=1,2,3)$ are given below;

$$
\begin{aligned}
& R_{1, e}=\left(\frac{1}{2}\right) f_{r}^{(m)} f_{e}^{\prime \prime(m)}-\left(\frac{1}{2}\right) \zeta\left(f_{e}^{\prime(m)} \frac{\partial f_{e}^{\prime(m)}}{\partial \zeta}-f_{e}^{\prime \prime(m)} \frac{\partial f_{e}^{(m)}}{\partial \zeta}\right) \\
& R_{2, e}=\left(\frac{1}{2}\right) f_{e}^{(m)} \vartheta_{e}^{\prime(m)}+N_{t} \vartheta_{e}^{\prime 2(m)}+E c\left(1+\frac{1}{\beta}\right) f_{e}^{\prime \prime 2(m)}+N_{b} \vartheta_{e}^{\prime(m)} \varphi_{e}^{\prime(m)} \\
& -\left(\frac{1}{2}\right) \zeta\left(f_{e}^{\prime(m)} \frac{\partial \vartheta_{e}^{(m)}}{\partial \zeta}-\vartheta_{e}^{\prime(m)} \frac{\partial f_{e}^{(m)}}{\partial \zeta}\right) \\
& R_{3, e}=(1 / 2) L e f_{e}^{(m)} \varphi_{e}^{\prime(m)}-(1 / 2) L e \zeta\left(f_{e}^{\prime(m)} \frac{\partial \varphi_{e}^{(m)}}{\partial \zeta}-\varphi_{e}^{\prime(m)} \frac{\partial f_{e}^{(m)}}{\partial \zeta}\right) .
\end{aligned}
$$

\section{Collocation}

The interval $\zeta \in \Lambda_{m} \equiv\left[\zeta_{m-1}, \zeta_{m}\right]$ is transformed to $s \in[-1,1]$ using the linear transformation;

$\zeta=\frac{1}{2}\left(\zeta_{m}-\zeta_{m-1}\right) s+\frac{1}{2}\left(\zeta_{m}+\zeta_{m-1}\right)$

and $\eta \in[0, \infty]$ is transformed to $x \in[-1,1]$ using;

$\eta=\frac{1}{2} C(x+1)$

where $C$ is a number large enough to represent the condition at infinity. For collocation, we use the Gauss-Lobatto collocation points, defined as;

$x_{i}=\cos \left(\frac{\pi i}{N_{x}}\right), \quad s_{j}=\cos \left(\frac{\pi j}{N_{s}}\right), \quad i=0,1, \ldots, N_{x}, \quad j=0,1, \ldots, N_{s}$.

The PDE is then arranged into matrix form using the Chebyshev differentiation matrix as defined in [28], where $\mathrm{D}$ denotes differentiation with respect to $\eta$ and denotes differentiation with respect to $\zeta$. If we denote the order of the differential by $n$, then the general form can be expressed as;

$$
\begin{aligned}
& \left.\frac{\partial^{n} f^{(m)}}{\partial x^{n}}\right|_{\left(s_{j}, x_{i}\right)}=\mathbf{D}^{n} F_{i}^{(m)},\left.\quad \frac{\partial f^{(m)}}{\partial s}\right|_{\left(s_{j}, x_{i}\right)}=\sum_{q=0}^{N s} \mathbf{d}_{i q}^{(m)} F_{q}^{(m)},\left.\frac{\partial^{n} \vartheta^{(m)}}{\partial x^{n}}\right|_{\left(s_{j}, x_{i}\right)}=\mathbf{D}^{n} T_{i}^{(m)},\left.\quad \frac{\partial \vartheta^{(m)}}{\partial s}\right|_{\left(s_{j}, x_{i}\right)}= \\
& \sum_{q=0}^{N s} \mathbf{d}_{i q}^{(m)} T_{q}^{(m)},\left.\frac{\partial^{n} \varphi^{(m)}}{\partial x^{n}}\right|_{\left(s_{j}, x_{i}\right)}=\mathbf{D}^{n} P_{i}^{(m)},\left.\quad \frac{\partial \varphi^{(m)}}{\partial s}\right|_{\left(s_{j}, x_{i}\right)}=\sum_{q=0}^{N s} \mathbf{d}_{i q}^{(m)} P_{q}^{(m)}, \quad i=0,1, \ldots, N x,
\end{aligned}
$$

where

$$
\begin{aligned}
& F_{i}=\left[f\left(s_{j}, x_{0}\right), f\left(s_{j}, x_{1}\right), \ldots, f\left(s_{j}, x_{N x}\right)\right]^{T}, \quad T_{i}=\left[\vartheta\left(s_{j}, x_{0}\right), \vartheta\left(s_{j}, x_{1}\right), \ldots, \vartheta\left(s_{j}, x_{N x}\right)\right]^{T}, \\
& P_{i}=\left[\varphi\left(s_{j}, x_{0}\right), \varphi\left(s_{j}, x_{1}\right), \ldots, \varphi\left(s_{j}, x_{N x}\right)\right]^{T}, \quad \mathbf{d}=\frac{2 d}{\zeta_{m}-\zeta_{m-1}}, \quad \mathbf{D}=\frac{2 D}{C} .
\end{aligned}
$$

Applying Eq. (26) to Eq. (11) we obtain; 
http://wjst.wu.ac.th

$$
\left(a_{0, e} \mathbf{D}^{3}+\mathbf{a}_{\mathbf{1 , e}} \mathbf{D}^{2}+\mathbf{a}_{\mathbf{2}, \mathbf{e}} \mathbf{D}+\mathbf{a}_{\mathbf{3}, \mathbf{e}} I+\mathbf{a}_{\mathbf{4}, \mathbf{e}} \mathbf{d}_{i i}^{(m)}(\mathbf{D})+\mathbf{a}_{\mathbf{5}, \mathbf{e}} \mathbf{d}_{i i}^{(m)} I\right) F_{i, e+1}^{(m)}+\mathbf{a}_{\mathbf{4}, \mathbf{e}} \sum_{\substack{q=0 \\ q \neq i}}^{N s-1} \mathbf{d}_{i q}^{(m)}(\mathbf{D}) F_{q, e+1}^{(m)}
$$

$+\mathbf{a}_{\mathbf{5}, \mathbf{e}} \sum_{\substack{q=0 \\ q \neq i}}^{N S-1} \mathbf{d}_{i q}^{(m)} F_{q, e+1}^{(m)}+a_{6, e} T_{i, e+1}^{(m)}+a_{7, e} P_{i, e+1}^{(m)}=R R_{1, e}$,

$\left(b_{0, e} \mathbf{D}^{2}+\mathbf{b}_{\mathbf{1}, \mathbf{e}} \mathbf{D}+b_{2, e} I+\mathbf{b}_{\mathbf{3}, \mathbf{e}} \mathbf{d}_{i i}^{(m)} I\right) T_{i, e+1}^{(m)}+\mathbf{b}_{\mathbf{3}, \mathbf{e}} \sum_{q=0}^{N S-1} \mathbf{d}_{i q}^{(m)} T_{q, e+1}^{(m)}+\left(\mathbf{b}_{\mathbf{4}, \mathbf{e}} \mathbf{D}^{2}+\mathbf{b}_{\mathbf{5}, \mathbf{e}} \mathbf{D}+\mathbf{b}_{\mathbf{6}, \mathbf{e}} I+\right.$

b7,ed $i(m) I F i, e+1(m)+\mathbf{b} 7, \mathbf{e} q=0 q \neq i N s-1 \mathbf{d} i q(m) F q, e+1(m)+b 8, e \mathbf{D} 2+\mathbf{b} \mathbf{9}, \mathbf{e D} P i, e+1(m)=R R 2, e$, $\left(c_{0, e} \mathbf{D}^{2}+\mathbf{c}_{\mathbf{1}, \mathbf{e}} \mathbf{D}+c_{2, e} I+\mathbf{c}_{\mathbf{3}, \mathbf{e}} \mathbf{d}_{i i}^{(m)}\right) P_{i, e+1}^{(m)}+\mathbf{c}_{3, \mathbf{e}} \sum_{\substack{q=0 \\ q \neq i}}^{N S-1} \mathbf{d}_{i q}^{(m)} P_{q, e+1}^{(m)}+\left(\mathbf{c}_{\mathbf{4}, \mathbf{e}} \mathbf{D}+\mathbf{c}_{\mathbf{5}, \mathbf{e}} I+\mathbf{c}_{\mathbf{6 , e}} \mathbf{d}_{i i}^{(m)} I\right) F_{i, e+1}^{(m)}+$ $\mathbf{c}_{\mathbf{6}, \mathbf{e}} \sum_{q=0}^{N s-1} \mathbf{d}_{i q}^{(m)} F_{q, e+1}^{(m)}+c_{7, e} \mathbf{D}^{2} T_{i, e+1}^{(m)}=R R_{3, e}$,

where the bold variable coefficients represent diagonal matrices;

$$
\begin{aligned}
& R R_{1, e}=R_{1, e}-\left(\mathbf{a}_{\mathbf{4}, \mathbf{e}} \mathbf{d}_{i N s}^{(m)}(\mathbf{D})+\mathbf{a}_{\mathbf{5}, \mathbf{e}} \mathbf{d}_{i N s}^{(m)} I\right) F_{N_{s}, e}^{(m)} \\
& R R_{2, e}=R_{2, e}-\mathbf{b}_{3, \mathbf{e}} \mathbf{d}_{i N s}^{(m)} T_{N_{s}, r}^{(m)}-\mathbf{b}_{7, \mathbf{e}} \mathbf{d}_{i N s}^{(m)} F_{N_{s}, e}^{(m)}, \quad \text { and } \\
& R R_{3, e}=R_{3, e}-\mathbf{c}_{3, \mathbf{e}} \mathbf{d}_{i N s}^{(m)} P_{N_{S}, r}^{(m)}-\mathbf{c}_{\mathbf{6}, \mathbf{e}} \mathbf{d}_{i N s}^{(m)} F_{N_{S}, e}^{(m)}
\end{aligned}
$$

and $\mathrm{I}$ is an $(N x+1) \times(N x+1)$ identity matrix. The equation is then expressed in matrix form as;

$$
\left[\begin{array}{lll}
A 11_{i, j} & A 12_{i, j} & A 13_{i, j} \\
A 21_{i, j} & A 22_{i, j} & A 23_{i, j} \\
A 31_{i, j} & A 32_{i, j} & A 33_{i, j}
\end{array}\right]\left[\begin{array}{c}
F_{i}^{(m)} \\
T_{i}^{(m)} \\
P_{i}^{(m)}
\end{array}\right]=\left[\begin{array}{c}
R R_{1, e}^{(m)} \\
R R_{2, e}^{(m)} \\
R R_{3, e}^{(m)}
\end{array}\right]
$$

where

$$
\begin{aligned}
& A 11_{i, i}=a_{0, e} \mathbf{D}^{3}+\mathbf{a}_{\mathbf{1}, \mathbf{e}} \mathbf{D}^{2}+\mathbf{a}_{2, \mathbf{e}} \mathbf{D}+\mathbf{a}_{\mathbf{3}, \mathbf{e}} I+\mathbf{a}_{\mathbf{4}, \mathbf{e}} \mathbf{d}_{i i}^{(m)}(\mathbf{D})+\mathbf{a}_{\mathbf{5 , e}} \mathbf{d}_{i i}^{(m)} I, \\
& A 11_{i, j}=\mathbf{a}_{\mathbf{4}, \mathbf{e}} \mathbf{d}_{i j}^{(m)}(\mathbf{D})+\mathbf{a}_{\mathbf{5}, \mathbf{e}} \mathbf{d}_{i j}^{(m)} I, A 12_{i, i}=a_{6, e} I, A 13_{i, i}=a_{7, e} I, \quad A 12_{i, j}=A 13_{i, j}=\mathbf{0}, \\
& A 21_{i, i}=\mathbf{b}_{\mathbf{4}, \mathbf{e}} \mathbf{D}^{2}+\mathbf{b}_{\mathbf{5}, \mathbf{e}} \mathbf{D}+\mathbf{b}_{\mathbf{6}, \mathbf{e}} I+\mathbf{b}_{7, \mathbf{e}} \mathbf{d}_{i i}^{(m)} I, A 21_{i, j}=\mathbf{b}_{7, \mathbf{e}} \mathbf{d}_{i j}^{(m)} I, \\
& A 22_{i, i}=b_{0, e} \mathbf{\mathbf { D } ^ { 2 }}+\mathbf{b}_{\mathbf{1}, \mathbf{e}} \mathbf{D}+b_{2, e} I+\mathbf{b}_{\mathbf{3}, \mathbf{e}} \mathbf{d}_{i i}^{(m)} I, A 22_{i, j}=\mathbf{b}_{\mathbf{3}, \mathbf{e}} \mathbf{d}_{i j}^{(m)} I, \\
& A 23_{i, i}=b_{8, e} \mathbf{D}^{2}+\mathbf{b}_{\mathbf{9}, \mathbf{e}} \mathbf{D}, A 23_{i, j}=\mathbf{0}, \\
& A 31_{i, i}=\mathbf{c}_{\mathbf{4}, \mathbf{e}} \mathbf{D}+\mathbf{c}_{\mathbf{5}, \mathbf{e}} I+\mathbf{c}_{\mathbf{6}, \mathbf{e}} \mathbf{d}_{i i}^{(m)} I, \quad A 31_{i, j}=\mathbf{c}_{\mathbf{6}, \mathbf{e}} \mathbf{d}_{i j}^{(m)} I, \quad A 32_{i, i}=c_{7, e} \mathbf{D}^{2}, A 32_{i, j}=\mathbf{0} \\
& A 33_{i, i}=c_{0, e} \mathbf{D}^{2}+\mathbf{c}_{\mathbf{1 , e}} \mathbf{D}+c_{2, e} I+\mathbf{c}_{3, \mathbf{e}} \mathbf{d}_{i i}^{(m)} I, A 33_{i, j}=\mathbf{c}_{\mathbf{3}, \mathbf{e}} \mathbf{d}_{i j}^{(m)} I .
\end{aligned}
$$

Here, $A_{r s}(i, i)$ is the diagonal of each $A_{r s}(i, j)$ matrix, where $(r=s=1,2,3)$.

\section{Results and discussion}

The governing equations in this study were solved using the multi-domain bivariate spectral quasi-linearization method, as described above. The equations were solved in the interval $\zeta \in[0,40]$, which was divided into 40 non-overlapping sub-intervals. 
http://wjst.wu.ac.th

Table 1 Comparison of result for $-\boldsymbol{\vartheta}^{\prime}(\mathbf{0})$ with those of existing literature for various values of $\boldsymbol{P r}$ when $\boldsymbol{\beta} \rightarrow \infty, f w=0, \lambda=0, N_{1}=0, E c=0, D f=0, L e=0, S r=0, H e=0$, and $R=0$, in the absence of nanoparticles.

\begin{tabular}{cccc}
\hline $\boldsymbol{P r}$ & Present result & $\boldsymbol{r e f}([29])$ & $\operatorname{ref}([30])$ \\
\hline 0.7 & 0.35082 & 0.3508 & 0.35005 \\
1 & 0.44387 & - & 0.44403 \\
2 & 0.68310 & 0.6831 & 0.68316 \\
7 & 1.38635 & - & 1.38625 \\
10 & 1.67916 & 1.6808 & 1.68011 \\
\hline
\end{tabular}

Table 2 Table of values showing values of $\boldsymbol{C} \boldsymbol{f}_{\boldsymbol{x}}$ and $\boldsymbol{N} \boldsymbol{u}_{\boldsymbol{x}}$ for large values of $\boldsymbol{\zeta}$.

\begin{tabular}{ccc}
\hline$\zeta$ & $C f x$ & $N u_{x}$ \\
\hline 5 & 1.28518451 & 0.31579130 \\
10 & 1.30437685 & 0.26738413 \\
15 & 1.34130402 & 0.18237793 \\
20 & 1.39733684 & 0.05400245 \\
25 & 1.47642042 & -0.12921402 \\
30 & 1.58476582 & -0.38595703 \\
35 & 1.73157090 & -0.74660041 \\
40 & 1.93019264 & -1.26105318 \\
\hline
\end{tabular}

Table 1 shows the comparison of the present research result for the Prandtl number $\mathrm{Pr}$ with those of Soundalgekar and Murty [29] and Patil et al. [30]. The table displays values of the Nusselt number for different values of $\mathrm{Pr}$. A good agreement is achieved. We note also that the heat transfer coefficient increases as the $\operatorname{Pr}$ number increases.

Table 2 shows the skin friction coefficient and Nusselt number for different values of $\zeta$. The mass transfer is not considered here due to the fact that we have assumed the nanoparticle flux to vanish at the boundary. This point was noted by Kuznetsov and Nield [16], who pointed out that the dimensionless mass flux, represented by the Sherwood number, is zero at this point. We note that, as we approach $\zeta$, the skin friction coefficient increases, while the reduced Nusselt number decreases. This shows that the non-similarity parameter has an effect on the fluid flow and heat transfer characteristics, even at large $\zeta$. 


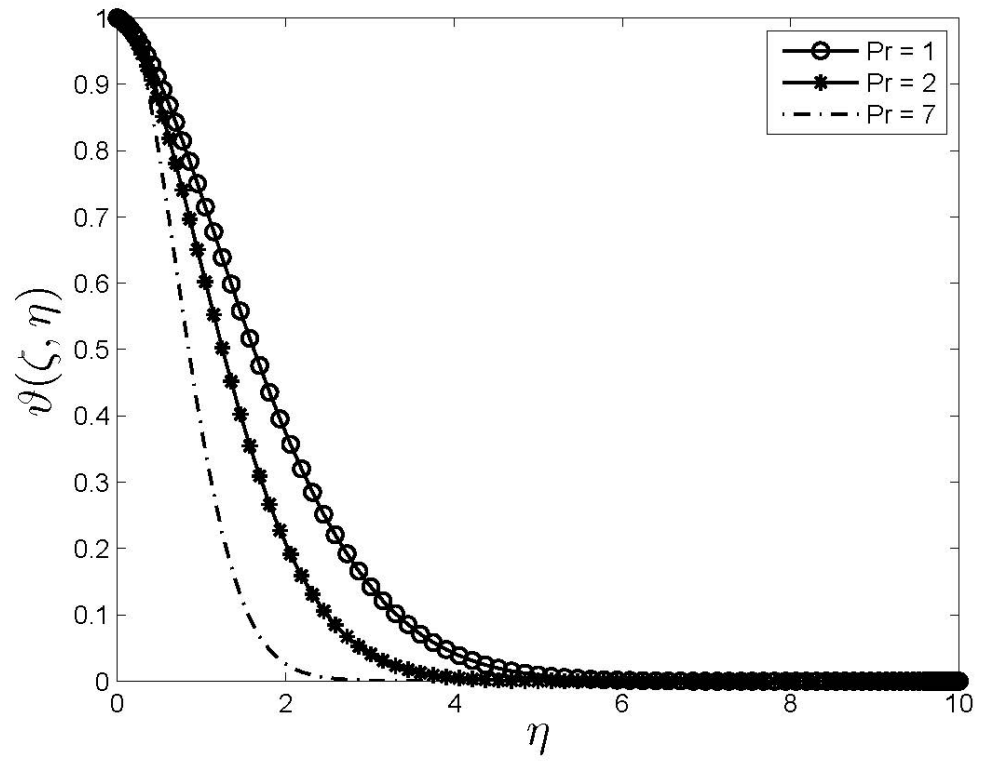

Figure 2 Effect of Prandtl number $\operatorname{Pr}$ on temperature profile $\vartheta(\zeta, \eta)$ when $r=0.5, N_{t}=0.1, N_{b}=$ $0.5, f w=1, \beta=2, \lambda=0.5, N_{1}=0.5, E c=1, D_{f}=0.1, L e=5, S r=0.1, H e=0.001$, and $R=0.5$.

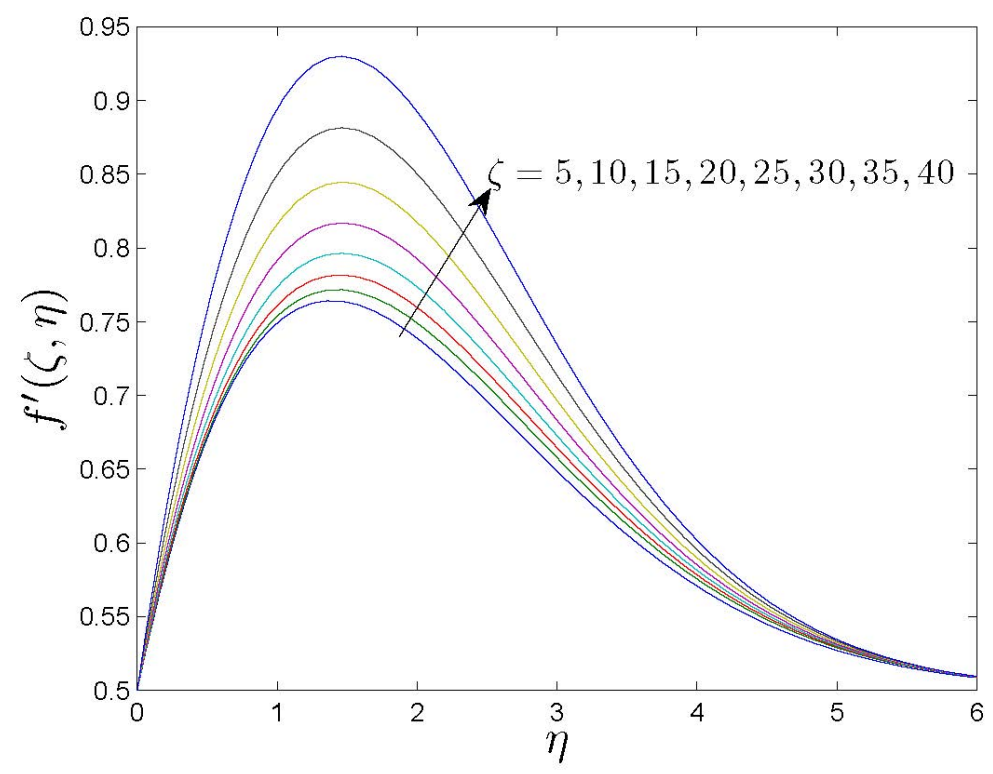

Figure 3 Velocity profile at different values of $\zeta$ when $\operatorname{Pr}=0.7, r=0.5, N_{t}=0.1, N_{b}=0.5$, $f w=1, \beta=2, \lambda=0.5, N_{1}=0.5, E c=1, D_{f}=0.1, L e=5, S r=0.1, H e=0.001$, and $R=0.5$. 


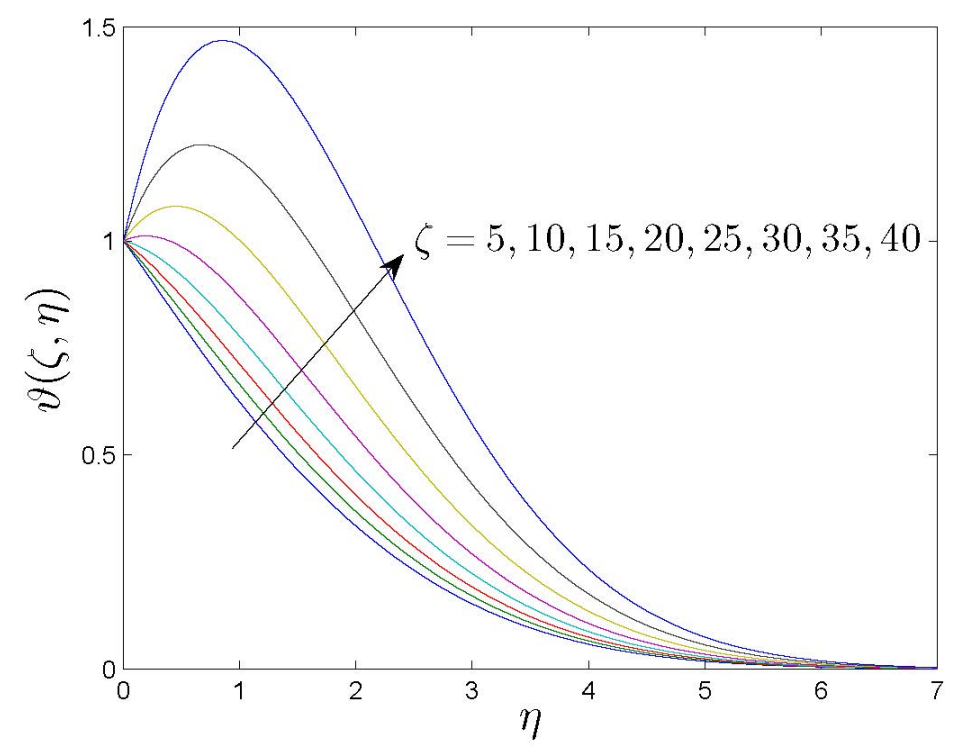

Figure 4 Temperature profile at different values of $\zeta$ when $r=0.5, N_{t}=0.1, N_{b}=0.5, f w=1$, $\beta=1, \lambda=0.5, N_{1}=0.5, E c=0.1, D_{f}=0.1, L e=1, S r=0.1, H e=0.001$, and $R=0.3$.

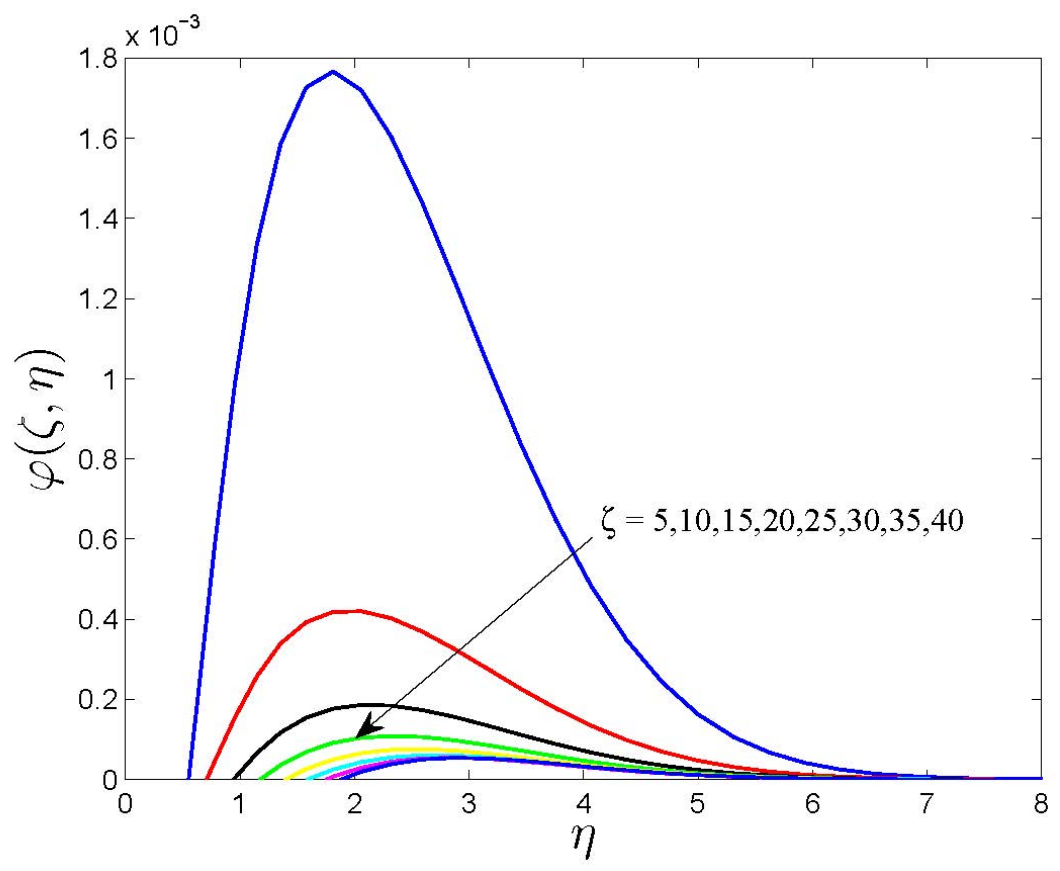

Figure 5 Concentration profile at different values of $\zeta$ when $r=0.5, N_{t}=0.1, N_{b}=0.5, f w=1$, $\beta=1, \lambda=0.5, N_{1}=0.5, E c=0.1, D_{f}=0.1, L e=1, S r=0.1, H e=0.001$, and $R=0.3$. 
http://wjst.wu.ac.th

Figure 2 shows the effect of the Prandtl number $\operatorname{Pr}$ on the boundary layer temperature profiles. The Prandtl number is the ratio of viscosity to diffusivity, and thus gives an insight into the physics of the fluid. In this particular problem, increasing the Prandtl number reduces the temperature profiles so that the thermal boundary layer thickness becomes thinner. The reduction is due to the increase in the viscosity, which in turn enhances the momentum boundary layer thickness. This attribute of $P r$ is similar to the observations in Soundalgekar and Murty [29].

Figures 3 - 5 give a visual display of the velocity, temperature and concentration profiles for different $\zeta$. In Figure 3, we note that there is an increase in the velocity profiles with $\zeta$. This implies a local maximum value of the velocity profile in the boundary layer region when $\zeta$ is increased. The same trend is obtainable for the temperature profiles in Figure 4, while in Figure 5, the concentration profiles are reduced with an increase in $\zeta$.

In Figures 6 and 7, we show the effect of the Casson parameter on the velocity and temperature profiles. When Casson parameter is increased, the yield stress is suppressed and the velocity reduces, but in the scenario observed in Figure 6, there is an enhancement of the velocity profiles near the wall, while away from the wall it is reduced. This suggests that the resistance to the flow as a result of an increase in the Casson parameter is reduced closer to the wall, and at this point the yield stress is not suppressed. The momentum boundary layer thickness is initially enhanced at the wall, but away from the wall it is reduced. In Figure 6 there is a decrease in the temperature profiles as the Casson parameter is increased; the yield stress is suppressed here as well, and the fluid temperature is reduced, thereby leading to a decrease in the thermal boundary layer thickness. This trend was also observed in [31].

Figures 8 and 9 depict temperature profiles for the heat generation $H e$ and viscous dissipation $E c$ parameters. We note that the temperature profiles are enhanced when $\mathrm{He}$ is increased. The effect of heat generation parameter is to release heat energy to the fluid flow. This energy release increases the temperature profiles as the parameter values are increased, and thus lead to an increase in the thermal boundary layer thickness. In Figure 9, we observe that increasing $E c$ also increases the temperature profiles. The viscous dissipation parameter measures heat production due to friction and viscous heating. As expected, this produces an increase in the temperature profile and thermal boundary layer thickness.

We show the effect of thermophoresis parameter $N_{t}$ on the temperature profile in Figure 10. Thermophoresis is associated with the movement of nanoparticles from a hot wall to a cold wall, and because this movement is generated by temperature gradients, this creates a fast flow away from the moving plate. Thus more fluid is heated away from the surface and this leads to an increase in the temperature within the boundary layer. The nanoparticle concentration profiles are negative at the wall for all parameters considered. This is due to the nanoparticle flux condition at the boundary, and this outcome is consistent with recent studies, see [16,18].

The effect of the thermophoresis parameter $N_{t}$ on the concentration profile is shown in Figure 11. As $N_{t}$ increases, the concentration profiles increase as well. This is due to the fact that higher thermophoresis values suggest a higher concentration of the nanoparticles in the fluid, hence increasing the concentration boundary layer thickness. It was observed that there is no significant difference in varying the values of the Brownian motion with the temperature profile. As suggested by Khan et al. [18], this point is understood better when we substitute $\varphi^{\prime}(\zeta, 0)=-(N t / N b) \vartheta^{\prime}(\zeta, 0)$ into the dimensionless energy Eq. (12). The thermophoresis term ideally goes to zero, thus, the alteration in temperature profile for different values of $N_{b}$ is inconsequential [16].

Figure 12 shows the effect of the Brownian motion parameter $N_{b}$ on the concentration profiles. It was observed that the concentration profiles decrease with $N_{b}$.

Figures 13 and 14 show the effects of the Dufour number and the Soret number on the temperature and concentration profiles, respectively. In Figure 13, we note how the thermal energy flux is affected by the concentration gradients, due to the decrease in the temperature profile. The effect of the temperature gradient is reduced and, thus, there is a cooling of the boundary layer region. In effect, this implies the thermal boundary layer thickness is decreased as the parameter is increased. The effect of the Soret number can be observed in Figure 14. The Soret number is the ratio of thermal diffusion coefficient and diffusion coefficient. This ratio represents the mass flux produced by temperature gradients. As the Soret number 
http://wjst.wu.ac.th

increases, the concentration profiles are also enhanced. This suggests that the temperature gradients produce a mass flux which enhances the concentration profiles and, hence, increases the nanoparticle concentration boundary layer thickness.

Figure 15 shows the effect of the chemical reaction parameter $R$ on the concentration profiles. It is observed that increasing $R$ decreases the concentration profiles. This implies that higher values of $R$ lead to a decrease in the chemical molecular diffusivity, causing a decrease in the concentration of the diffusing species and reduction in mass diffusion, and this effect leads to the thinning of the concentration boundary layer.

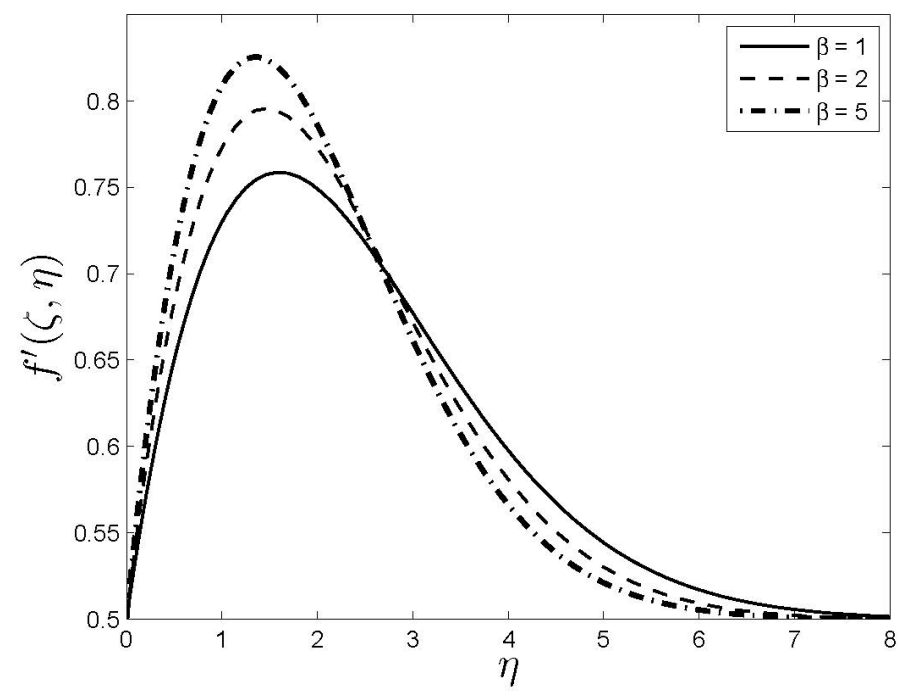

Figure 6 Effect of Casson parameter $\beta$ on velocity profile $f^{\prime}(\zeta, \eta)$ when $\operatorname{Pr}=0.7, r=0.5, N_{t}=0.1$, $N_{b}=0.5, f w=1, \lambda=0.5, N_{1}=0.5, E c=1, D_{f}=0.1, L e=5, S r=0.1, H e=0.001$, and $R=0.5$.

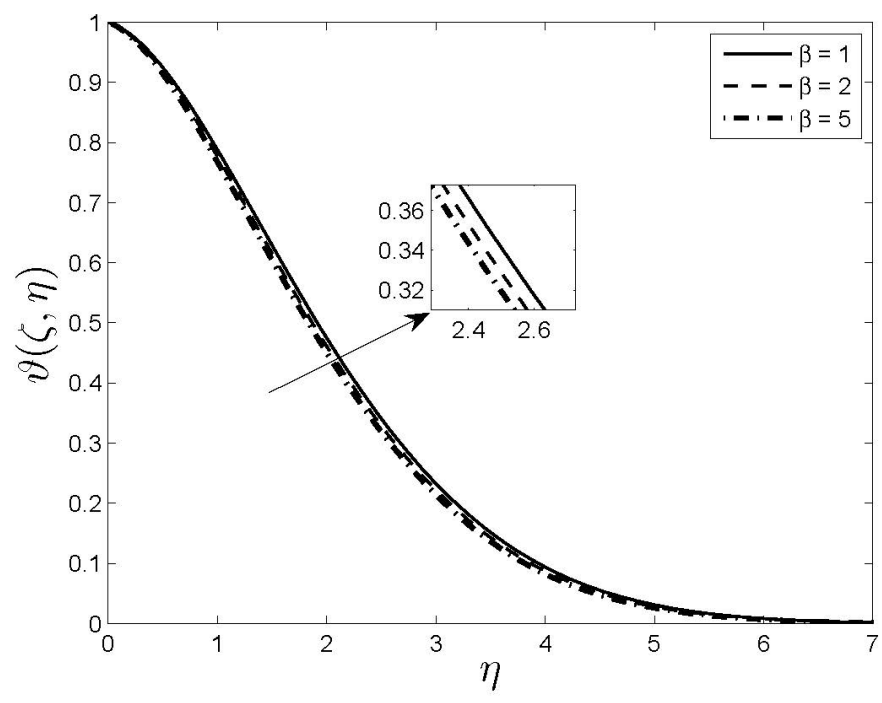

Figure 7 Effect of Casson parameter $\beta$ on temperature profile $\vartheta(\zeta, \eta)$ when $\operatorname{Pr}=0.7, r=0.5$, $N_{t}=0.1, N_{b}=0.5, f w=1, \lambda=0.5, N_{1}=0.5, E c=1, D_{f}=0.1, L e=5, S r=0.1$, He = 0.001, and $R=0.5$. 


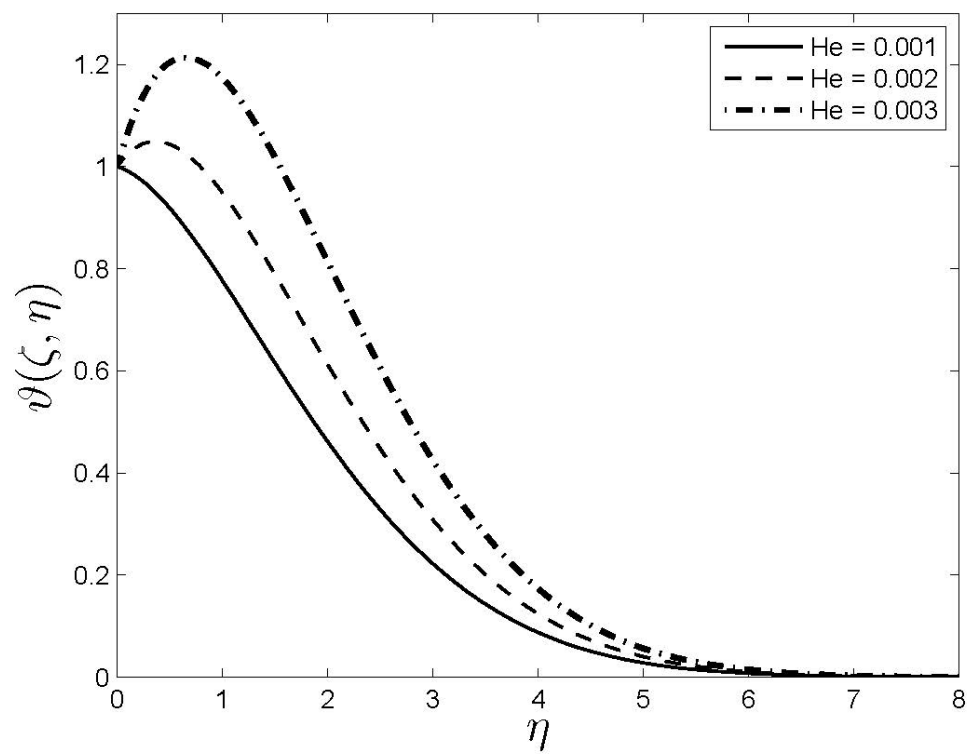

Figure 8 Effect of heat generation parameter $H e$ on temperature profile $\vartheta(\zeta, \eta)$ when $\operatorname{Pr}=0.7$, $r=0.5, N_{t}=0.1, N_{b}=0.5, f w=1, \beta=1, \lambda=0.5, N_{1}=0.5, E c=1, D_{f}=0.1, \operatorname{Le}=5, S r=$ 0.1 , and $R=0.5$.

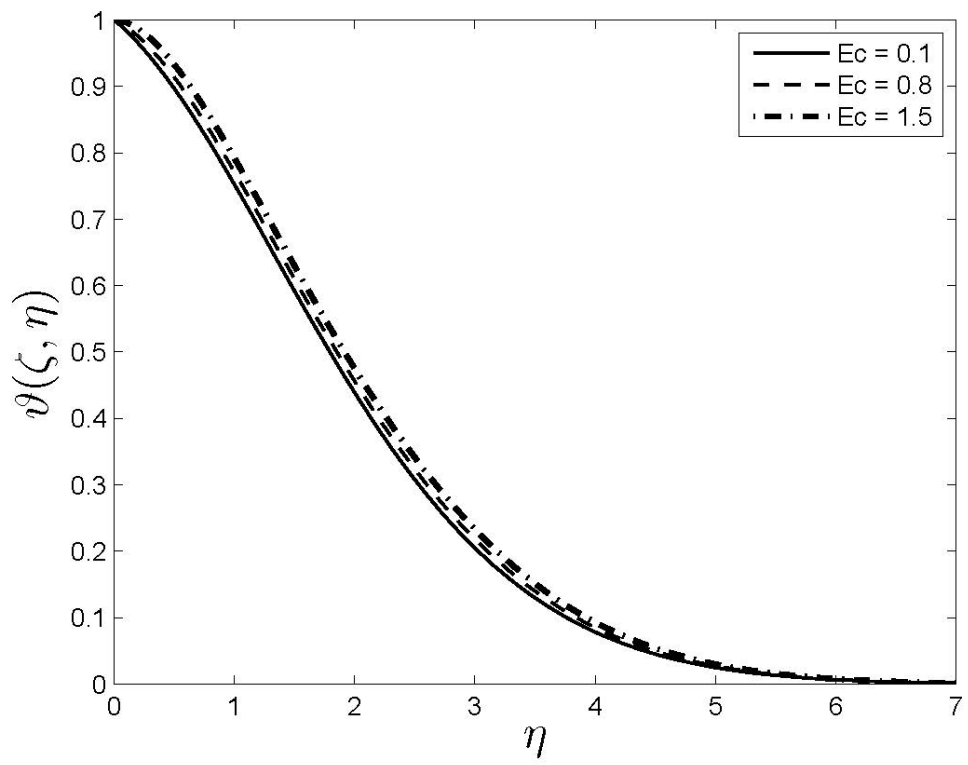

Figure 9 Effect of viscous dissipation parameter $E c$ on temperature profile $\vartheta(\zeta, \eta)$ when $\operatorname{Pr}=0.7$, $r=0.5, N_{t}=0.1, N_{b}=0.5, f w=1, \beta=1, \lambda=0.5, N_{1}=0.5, D_{f}=0.1, L e=5, S r=0.1$, $H e=0.001$, and $R=0.5$. 


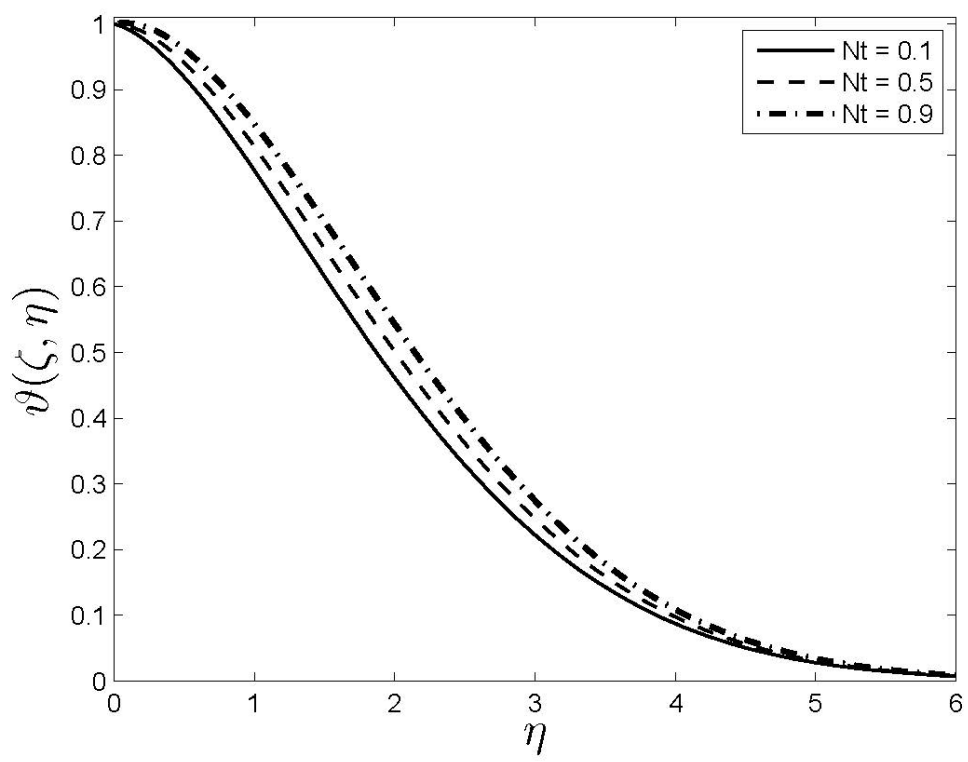

Figure 10 Effect of thermophoresis parameter $N_{t}$ on temperature profile $\vartheta(\zeta, \eta)$ when $\operatorname{Pr}=0.7$, $r=0.5, N_{b}=0.5, f w=1, \beta=1, \lambda=0.5, N_{1}=0.5, E c=1, D_{f}=0.1, L e=5, S r=0.1, H e=$ 0.001 , and $R=0.5$.

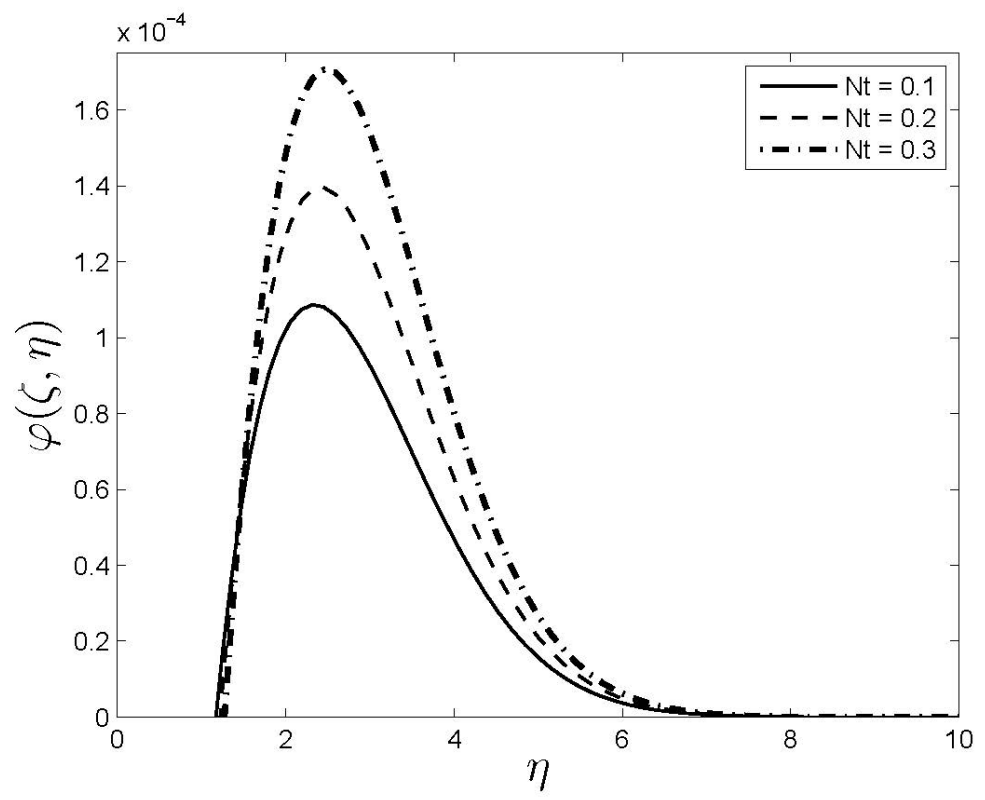

Figure 11 Effect of thermophoresis parameter $N_{t}$ on concentration profile $\varphi(\zeta, \eta)$ when $\operatorname{Pr}=0.7$, $r=0.5, N_{b}=0.5, f w=1, \beta=1, \lambda=0.5, N_{1}=0.5, E c=1, D_{f}=0.1, L e=5, S r=0.1, H e=$ 0.001 , and $R=0.5$. 


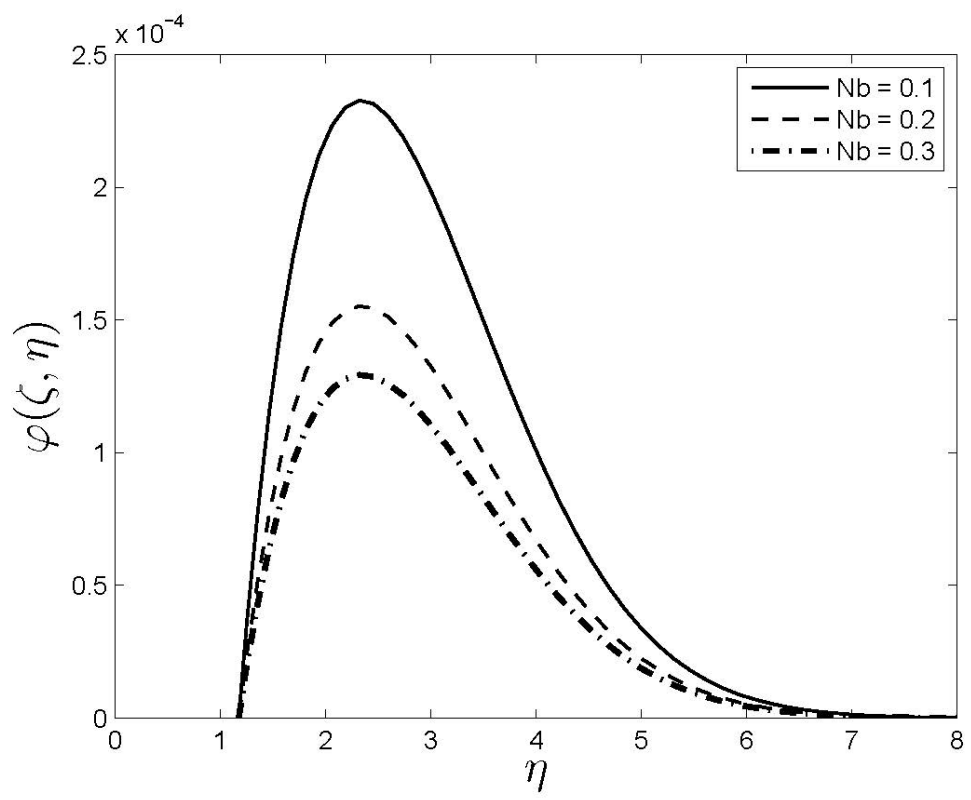

Figure 12 Effect of Brownian motion parameter $N_{b}$ on concentration profile $\varphi(\zeta, \eta)$ when $\operatorname{Pr}=0.7$, $r=0.5, N_{t}=0.1, f w=1, \beta=1, \lambda=0.5, N_{1}=0.5, E c=1, D_{f}=0.1, L e=5, S r=0.1, H e=$ 0.001 , and $R=0.5$.

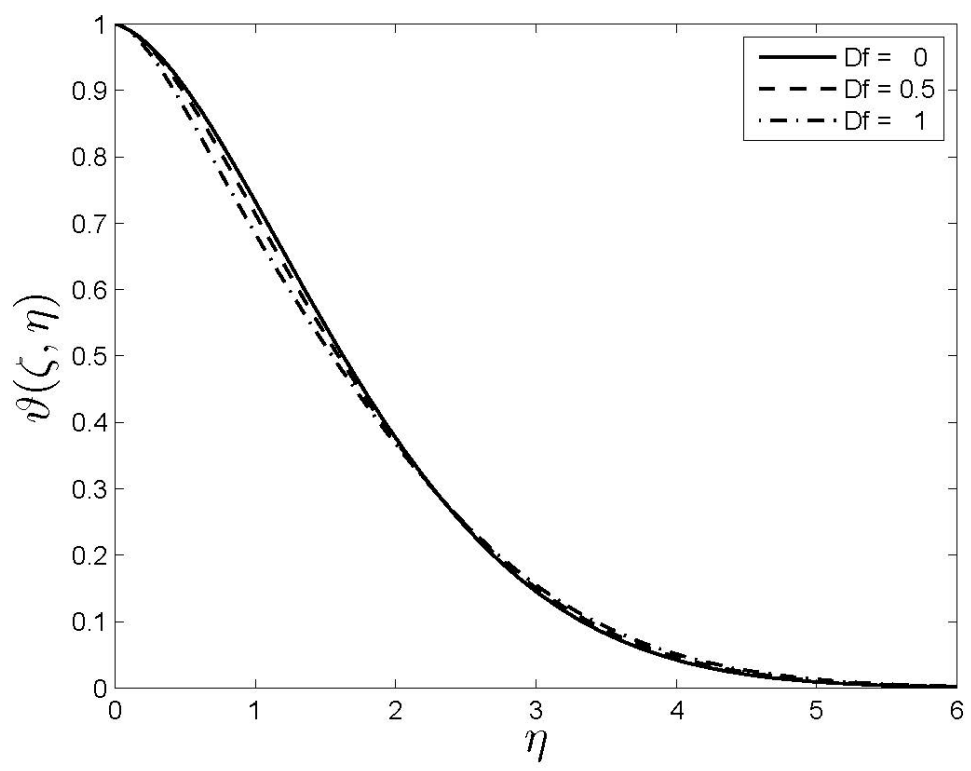

Figure 13 Effect of Dufour number $D_{f}$ on temperature profile $\vartheta(\zeta, \eta)$ when $\operatorname{Pr}=0.7, r=0.5$, $N_{t}=0.1, N_{b}=0.5, f w=1, \beta=1, \lambda=0.5, N_{1}=0.5, E c=1, L e=5, S r=0.1, H e=0.001$, and $R=0.5$. 


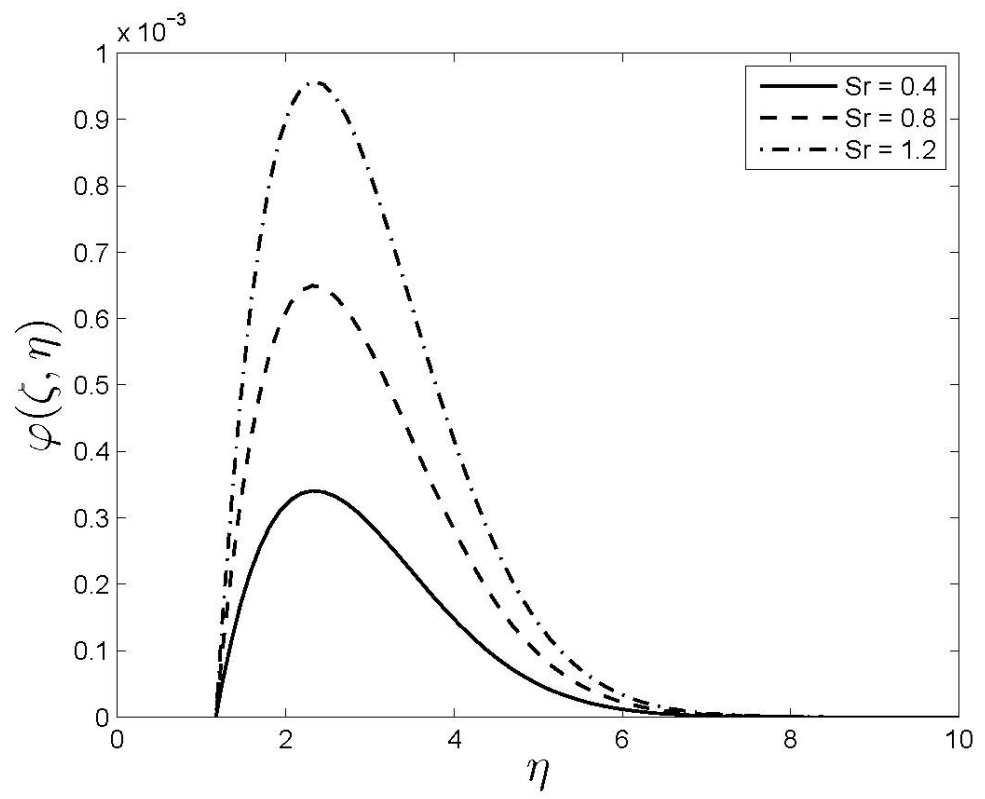

Figure 14 Effect of Soret number $\operatorname{Sr}$ on concentration profile $\varphi(\zeta, \eta)$ when $\operatorname{Pr}=0.7, r=0.5$, $N_{t}=0.1, N_{b}=0.5, f w=1, \beta=1, \lambda=0.5, N_{1}=0.5, E c=1, D_{f}=0.1, L e=5, H e=0.001$, and $R=0.5$.

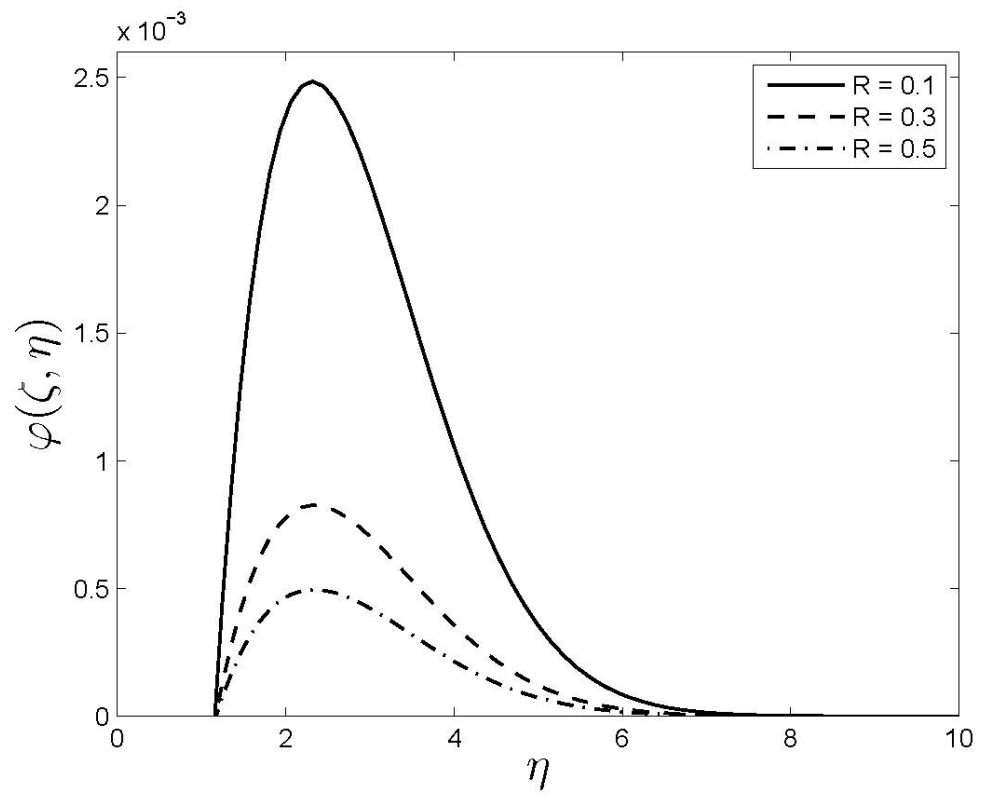

Figure 15 Effect of chemical reaction parameter $R$ on concentration profile $\varphi(\zeta, \eta)$ when $\operatorname{Pr}=0.7$, $r=0.5, N_{t}=0.1, N_{b}=0.5, f w=1, \beta=1, \lambda=0.5, N_{1}=0.5, E c=1, D_{f}=0.1, L e=5, S r=$ 0.1 , and $\mathrm{He}=0.001$. 
http://wjst.wu.ac.th

\section{Conclusions}

In this work, we have studied the boundary layer flow of a Casson nanofluid with a chemical reaction, heat generation, viscous dissipation, and thermo and thermal diffusion effects using the multi-domain bivariate spectral quasi-linearization method to solve the model equations. The qualitative influence of the different fluid parameters on the Casson fluid properties has been analyzed in detail. It was observed that the velocity boundary is enhanced by an increase in the non-similarity parameter; the thermal boundary increased with an increase in the Casson, heat generation, viscous dissipation and the thermophoresis parameters, while it decreased with an increase in the Dufour number.

We have showed that thermophoresis and the Soret parameter enhanced the nanoparticle concentration boundary, while the boundary was reduced by increasing the Brownian motion and the chemical reaction parameters.

\section{References}

[1] DD Joye, JP Bushinsky and PE Saylor. Mixed convection heat transfer at high Grashof number in a vertical tube. Ind. Eng. Chem. Res. 1989; 28, 1899-903.

[2] SPM Isa, NM Arifin and R Nazar. Mixed convection boundary layer flow of a Casson fluid near the stagnation point over a permeable surface. Malay. J. Fund. Appl. Sci. 2014; 10, 22-7.

[3] T Hayat, SA Shehzad, A Alsaedi and MS Alhothuali. Mixed convection stagnation point flow of Casson fluid with convective boundary conditions. Chin. Phys. Lett. 2012; 29, 114704.

[4] K Sharada and B Shankar. MHD mixed convection flow of a Casson fluid over an exponentially stretching surface with the effects of soret, dufour, thermal radiation and chemical reaction. World J. Mech. 2015; 5, 165-77.

[5] T Hayat, MB Ashraf, SA Shehzad, and A Alsaedi. Mixed convection flow of Casson nanofluid over a stretching sheet with convectively heated chemical reaction and heat source/sink. J. Appl. Fluid Mech. 2015; 8, 803-13.

[6] R Kandasamy, R Saravanan and KKS Prabhu. Chemical reaction on non-linear boundary layer flow over a porous wedge with variable stream conditions. Chem. Eng. Comm. 2010; 197, 522-43.

[7] G Makanda, S Shaw and P Sibanda. Effects of radiation on MHD free convection of a Casson fluid from a horizontal circular cylinder with partial slip in non-Darcy porous medium with viscous dissipation. Bound. Value Probl. 2015; 75, 1-14.

[8] M Aslefallah and D Rostamy. A numerical scheme for solving Space-Fractional equation by finite differences theta-method. Int. J. Adv. Appl. Math. Mech. 2014; 1, 1-9.

[9] M Aslefallah and D Rostamy. Numerical solution for Poisson fractional equation via finite differences theta-method. J. Math. Comput. Sci. 2014; 12, 132-42.

[10] M Aslefallah and E Shivanian. A nonlinear partial integro-differential equation arising in population dynamic via radial basis functions and theta-method. J. Math. Comput. Sci. 2014; 13, 14-25.

[11] M Aslefallah, D Rostamy and K Hosseinkhani. Solving time-fractional differential diffusion equation by theta-method. Int. J. Adv. Appl. Math. Mech. 2014; 2, 1-8.

[12] NG Kafoussias and EW Williams. Thermal-diffusion and diffusion-thermo effects on mixed free-forced convective and mass transfer boundary layer flow with temperature dependent viscosity. Int. J. Eng. Sci. 1995; 33, 1369-84.

[13] AJ Chamkha and SMM El-Kabeir. Unsteady heat and mass transfer by MHD mixed convection flow over an impulsively stretched vertical surface with chemical reaction and Soret and Dufour effects. Chem. Eng. Comm. 2013; 200, 1220-36.

[14] SUS Choi. Enhancing thermal conductivity of fluids with nanoparticles. ASME Publ. Fed. 1995; 231, 99-106.

[15] J Buongiorno. Convective transport in nanofluids. J. Heat Trans. 2006; 128, 240-50.

[16] AV Kuznetsov and DA Nield. Natural convective boundary layer flow of a nanofluid past a vertical plate: A revised model. Int. J. Therm Sci. 2014; 77, 126-9.

[17] M Fakour, A Vahabzadeh and DD Ganji. Scrutiny of mixed convection flow of a nanofluid in a 
http://wjst.wu.ac.th

vertical channel. Case Stud. Therm. Eng. 2014; 4, 15-23.

[18] JA Khan, M Mustafa, T Hayat and A Alsaedi. Three-dimensional flow of nanofluid over a non-linearly stretching sheet: An application to solar energy. Int. J. Heat Mass Trans. 2015; 86, 158-64.

[19] MY Malik, M Naseer, S Nadeem and A Rehman. The boundary layer flow of Casson nanofluid over a vertical exponentially stretching cylinder. Appl. Nanosci. 2014; 4, 869-73.

[20] S Nadeem, R Mehmood and NS Akbar. Optimized analytical solution for oblique flow of a Casson-nano fluid with convective boundary conditions. Int. J. Therm. Sci. 2014; 78, 90-100.

[21] UIR Haq, S Nadeem, ZH Khan and TG Okedayo. Convective heat transfer and MHD effects on Casson nanofluid flow over a shrinking sheet. Cent. Eur. J. Phys. 2014; 12, 862-71.

[22] MH Abolbashari, N Freidoonimehr, F Nazari and MM Rashidi. Analytical modeling of entropy generation for Casson nano-fluid flow induced by a stretching surface. Adv. Powder Tech. 2015; 26, 542-52.

[23] S Mukhopadhyay. Effects of thermal radiation on Casson fluid flow and heat transfer over an unsteady stretching surface subjected to suction/blowing. Chin. Phys. B 2013; 22, 114702.

[24] SS Motsa, P Dlamini and M Khumalo. A new multi-stage spectral relaxation method for solving chaotic initial value systems. Nonlin. Dyn. 2013; 72, 265-83.

[25] RE Bellman and RE Kalaba. Quasilinearization and nonlinear boundary-value problems. Soc. Ind. Appl. Math. Rev. 1966; 8, 401-2.

[26] SS Motsa, PG Dlamini and M Khumalo. Spectral relaxation method and spectral quasilinearization method for solving unsteady boundary layer flow problems. Adv. Math. Phys. 2014; 2014, 341964.

[27] SS Motsa, FG Awad, ZG Makukula and P Sibanda. The spectral homotopy analysis method extended to systems of partial differential equations. Abs. Appl. Anal. 2014; 2014, 241594.

[28] LN Trefethen. Spectral Methods in MATLAB. Society for Industrial and Applied Mathematics, 2000.

[29] VM Soundalgekar and R Murty. Heat transfer in flow past a continuous moving plate with variable temperature. Warme - und Stoffubertragung 1980; 14, 91-3.

[30] PM Patil, D Anikumar and S Roy. Unsteady thermal radiation mixed convection flow from a moving vertical plate in a parallel free stream: Effect of Newtonian heating. Int. J. Heat Mass Trans. 2013; 62, 534-40.

[31] T Hussain, SA Shehzad, A Alsaedi, T Hayat and M Ramzan. Flow of Casson nanofluid with viscous dissipation and convective conditions: A mathematical model. J. Central South Univ. 2015; 22, 1132-40. 